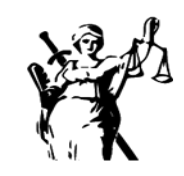

JUSTICIA

ISSN impreso 0124-7441
ISSN digital 2590-4566

\title{
Perspectiva sobre la calidad desde el seguimiento a graduados de la Facultad de Derecho de la Universidad San Buenaventura, Medellín
}

\author{
Perspective on quality from the follow-up to graduates of the \\ Faculty of Law of the San Buenaventura University, Medellín
}

Perpectiva sobre a qualidade da graduação e pós-graduação da facultade San Buenaventura Medellin

\author{
(iD) \\ Sonia Fátima Atehortúa Rengifo \\ Universidad San Buenaventura, Medellín, Colombia \\ asesora.th@usbmed.edu.co
}

Recibido: 14 de Noviembre de 2020 / Aceptado: 21 de julio de 2021

https://doi.org/10.17081/just.26.40.4336

\begin{abstract}
Resumen
Objetivo: El propósito principal de este artículo es establecer el perfil sociodemográfico, las competencias profesionales y la relación con la institución de los graduados de la Facultad de Derecho Universidad San Buenaventura, Medellín. Método: la población estuvo conformada por graduados del pregrado de Derecho, de la Especialización en Servicios Públicos Domiciliarios y de la Especialización en Ciencias Penales y Penitenciarias. La muestra fue de tipo no probabilístico con los graduados que decidieron participar voluntariamente. Se usó la técnica de grupos focales y se aplicaron encuestas para perfil sociodemográfico, competencias profesionales y participación institucional. También se hizo encuesta al director del programa de pregrado. Resultados: El número de mujeres graduadas es un poco más alto que el número de hombres; la gran mayoría de los graduados están empleados; la formación humanística es un sello particular de los graduados, las competencias en un segundo idioma son básicas; el vínculo con la institución es mínimo. Conclusiones: (i) Entre los principales hallazgos se destaca la correlación entre la formación de los graduados de derecho y la alta empleabilidad en el sector público y privado. (ii) Es conveniente imbricar distintos criterios y variables para estimar la calidad de la educación superior. (iii) Las reflexiones sobre el quehacer profesional y su relación con la sociedad son necesarias para impulsar acciones de mejoramiento.
\end{abstract}

PalABras ClaVes: Calidad de la educación, Universidad, graduados, competencia profesional.

\begin{abstract}
Tobjective: The main purpose of this article is to establish the socio-demographic profile, professional skills and relationship with the institution of the graduates of the San Buenaventura University Law School, Medellin. Methods: The population was made up of graduates of the undergraduate law program, the specialization in public home services and the specialization in criminal and prison sciences. The sample was non-probabilistic with the graduates who decided to participate voluntarily. The focus group technique was used and surveys were applied for socio-demographic profile, professional skills and institutional participation. The director of the undergraduate program was also surveyed. Results: The number of female
\end{abstract}


graduates is higher than the number of male graduates; the vast majority of graduates are employed; humanistic training is a particular hallmark of the graduates; skills in a second language are basic; the link with the institution is minimal. Conclusions: (i) Among the main findings is the correlation between the training of law graduates and high employability in the public and private sectors. (ii) Different criteria and variables should be interwoven to estimate the quality of higher education. (iii) Reflections on professional work and its relationship with society are necessary to promote improvement actions.

KEYWORDS: Quality of education, university, graduates, professional competence.

Como Citar: Atehortúa Rengifo, S. F. (2021). Perspectiva sobre la calidad desde el seguimiento a graduados de la Facultad de Derecho de la Universidad San Buenaventura, Medellín. Justicia, 26(40), 45-62. https://doi.org/10.17081/just.26.40.4336

\section{Introduction}

En el continuo proceso de mejoramiento, las Instituciones de Educación Superior, IES, también dirigen su mirada hacia los graduados. El seguimiento a los graduados y graduadas permite ver con detalle la relación entre los propósitos de la formación con el desenvolvimiento en el mundo exterior, de manera que se puedan implementar acciones en pos del mantenimiento y mejoramiento de la calidad de las instituciones. La Universidad San Buenaventura, Medellín, se propuso hacer seguimiento a los graduados de sus distintas facultades. En este trabajo se da cuenta particularmente de la participación en los distintos campos de actuación e influencia de los graduados de la Facultad de Derecho.

En el campo educativo, la calidad está asociada al desarrollo de competencias y habilidades, objetivos, pertinencia, cobertura, instalaciones, cuerpo docente, estándares, clasificaciones. Según Misas Arango (2004), "la calidad es un concepto socialmente construido. [...] La calidad es un concepto relativo, subjetivo y en proceso permanente de construcción. Tiene un carácter polisémico y multicontextual [...] "depende del capital cultural heredado por parte de los estudiantes que acceden a la universidad y de la calidad de las instituciones de educación media" (pp. 42-43). Por su parte, para Vázquez (2015) la calidad de la educación se relaciona principalmente con la noción de evaluación para medir resultados con instrumentos homogéneos, que desconocen las particularidades de los entornos y de los estudiantes y el proceso de enseñanza-aprendizaje. La razón de dicha inclinación radica en "la convicción neoliberal de que la tarea central de las escuelas no es la educación, sino la capacitación de los niños y jóvenes en ciertos desempeños de aplicación inmediata, ahora llamados competencias que deben ser empíricamente observables y cuantificables al igual que en la producción de cualquier mercancía" (p. 60).

A partir de estas dos posturas sobre la calidad, se puede tomar varios elementos. Uno de ellos es la necesidad de circunscribir la noción de la calidad en la educación a un tiempo y un espacio determinado, es decir, a un momento histórico particular; de este modo se puede ver hacia dónde se orientan los objetivos de la educación y cuáles son los medios para lograrlo. Otro punto es considerar el nivel de formación que se quiere evaluar; dentro de la misma educación superior hay diferencias considerables: los razonamientos sobre calidad en el pregrado son distintos a los que se requieren en el posgrado. "En el pregrado, un criterio de buena calidad es la formación que capacita para plantearse un problema y buscar soluciones alternativas [y por el contrario, un criterio de baja calidad es la formación] centrada exclusivamente en los procedimientos, en el trabajo estandarizado y normalizado" (Misas Arango, 2004, p.43). En el posgrado, nivel especialización, el criterio de calidad está más asociado con la capacidad de profundizar en un área específica. 
En lo que respecta propiamente a la Educación Superior Universitaria en Colombia, la calidad puede ser observada y monitoreada desde múltiples criterios, en distintas etapas del proceso formativo y con la consideración de los diversos actores relacionados con las instituciones: estudiantes, docentes, directivas, graduados, entre otros. Según del decreto 1330 de julio de 2019, expedido por el Ministerio de Educación, la calidad de los programas académicos de educación superior:

Es el conjunto de atributos articulados, interdependientes, dinámicos, construidos por la comunidad académica como referentes y que responden a las demandas sociales, culturales y ambientales. Dichos atributos permiten hacer valoraciones internas y externas a las instituciones, con el fin de promover su transformación y el desarrollo permanente de sus labores formativas, académicas, docentes, científicas, culturales y de extensión (Art. 2.5.3.2.1.1. D. 1330/2019)

Uno de estos ángulos desde donde se mira la calidad de la educación superior es desde los graduados, sujetos activos de la comunidad educativa, que pueden participar en la dirección de los establecimientos educativos (Art. 68. CPC), (Art. 6. L.115/1994). En los graduados confluyen de manera sincrónica el proceso y resultado de la formación recibida y las demandas y articulación con el sector productivo, los empleadores y, en general, de la sociedad civil. Dicho de otro modo, el graduado establece la primera línea estratégica del proceso de comunicación con el medio externo, de alguna manera el conocimiento creado se válida con su actuar en la sociedad. A partir de su experiencia y de la valoración de esa experiencia, se pueden construir reflexiones acerca de las cuestiones académicas y curriculares de cada programa académico, de los escenarios del desempeño laboral y de los problemas que se deben abordar en el mundo laboral, con el fin de dar respuesta a las demandas del entorno en materia de formación continua y avanzada (Constitución Política de Colombia, 2018, p.15).

De ahí que uno de los desafíos de la universidad con sus graduados es construir espacios de encuentro que permitan a la institucionalidad reconocerse y conocer el impacto real de las acciones académicas, para intervenir de forma adecuada en los distintos programas y estimular la agencia de los graduados en la construcción de una sociedad mejor. Además, la responsabilidad social universitaria involucra al graduado que la representa, pues su deber-ser como integrante de la comunidad académica no se agota con la titulación del profesional, sino que se extiende en las labores profesionales inscritas en su quehacer cotidiano. De ahí que sea necesario hacerles estudios de seguimiento, puesto que son "una de las estrategias más efectivas para retroalimentar la pertinencia de los programas académicos de las universidades de modo permanente, a fin de realizar los ajustes adecuados de acuerdo a las necesidades y problemáticas de la sociedad" (Berrún Castañón, 2015, p. 19).

La normatividad vigente exige a las IES la existencia de un programa de egresados, con el cual hacer seguimiento continuo de las apuestas institucionales y promover la participación de los graduados en la vida universitaria. Los graduados constituyen la impronta de la misión institucional y a través de ellos se puede articular las políticas institucionales que muestren realmente la inserción laboral o la actividad profesional de los graduados, en concordancia con lo estipulado recientemente por el Ministerio de Educación Nacional (D. 1330/2000).

El Observatorio Laboral para la Educación en Colombia, sistema adscrito al Ministerio de Educación, brinda datos e información a través de mapas y datos estadísticos a las IES, Gobierno, empleadores, entre otros, para reconocer el servicio de la educación a partir del rastreo a los graduados respecto a su situación laboral de acuerdo con su perfil profesional, lo cual incluye índices de empleabilidad, índice de ingresos y un panorama sobre los sectores en los que se ubican laboralmente. Si bien, es un instrumento útil para 
hacer seguimiento a los graduados, es necesario tener otras fuentes de información que profundicen en aspectos relacionados con las especificidades de los programas académicos y que den cuenta de aspectos de carácter más cualitativo, como la satisfacción de los graduados con su profesión, el cumplimiento de metas personales gracias al ejercicio de la profesión, el bienestar en el ejercicio profesional.

En el caso de los graduados de derecho, dentro del marco de la disciplina jurídica, están llamados a cumplir una función social progresiva conforme a la época y la realidad. La vida de las personas ha estado permeada por el derecho por cuanto incide en los cambios estructurales de la sociedad a través de la regulación normativa en asuntos de la vida cotidiana, que son transversales a los ámbitos económico, político, social, familiar. Por ello, se espera de los graduados de derecho un ejercicio profesional que facilite y garantice los medios de transformación social, moral y política de la sociedad.

La importancia de conocer el quehacer del graduado en derecho plantea retos para la toma de decisiones institucionales en relación con los programas, pertinencia del currículo, y permite fortalecer los procesos de autoevaluación institucional y del programa, en el marco de mejoramiento continuo.

La Facultad de Derecho de la Universidad San Buenaventura, Medellín, ofrece actualmente programas de formación en el pregrado de Derecho y en los posgrados de Especialización en Servicios Públicos Domiciliarios y Especialización en Derecho Procesal Constitucional. Al igual que las demás divisiones académicas de la Universidad, se rige por los principios franciscanos de solidaridad, justicia, fraternidad, y solidaridad, que son enseñados en sus currículos. Esto quiere decir que además de la formación en lo propiamente jurídico, hay una presencia muy fuerte de una serie de valores que singularizan el actuar profesional y laboral de los graduados. En este estudio participaron graduados del pregrado de Derecho, desde 1998, año de la primera cohorte; de la especialización en Servicios Públicos Domiciliarios, a partir del 2002-1, y de la Especialización en Ciencias Penales y Penitenciarias, a partir del 2010, hasta el 2018.

Los estudiantes de la Facultad de Derecho llevan a cabo actividades prácticas en el Consultorio Jurídico y Centro de Conciliación San Juan de Capistrano, adscrito a la Facultad. En este espacio, los estudiantes de los dos últimos años de carrera pueden señalar la solución a los problemas jurídicos expuestos por los usuarios de escasos recursos, "personas pobres" que acuden a su ayuda (L. 583/2000; C. Const. Sentencia C-110/2017).

La función del estudiante de los últimos años de la carrera es la de brindar atención, asesoría jurídica y representación de las "personas pobres" de la sociedad de manera gratuita, con el fin de resolver el problema planteado por el usuario ante el Consultorio Jurídico o del Centro de Conciliación según el caso con miras a la resolución del conflicto. Esta aproximación al problema jurídico desde estos espacios es el preámbulo del ejercicio profesional; una oportunidad para confirmar la vocación, tener "aprendizaje en contexto" (Osorio Valencia, 2017, p. 121) y "muestra de la creación de [esta] responsabilidad social, desde la carrera misma" (Fonseca Ferís, 2019, p.190). Es, sin dudas, un acercamiento a la vida laboral y al compromiso social que lleva implícita la profesión de abogado.

Para el graduado de un programa en pregrado o posgrado en derecho existe un conjunto de problemáticas que indefectiblemente se relacionan con tópicos como la familia, la propiedad, el delito, la justicia, el Estado, entre otras, que exigen la reconfiguración de la enseñanza de acuerdo con la comprensión y crítica de dichas instituciones y conceptualizaciones. De ahí que se busque proponer a los estudiantes una metodología didáctica específica basada en problemas y estudios de caso que le permitan elaborar un proceso hermenéutico y crítico respecto a la experiencia de las ciudadanías, antes de la práctica profesional en los consultorios jurídicos y centros de conciliación. 
Uno de los retos de la Facultad de Derecho, actualmente, es considerar otras formas de educación jurídica que se orienten a formar personas que buscan la justicia social, en lugar de abogados que entorpezcan el ejercicio legítimo de la defensa de los derechos de las personas en su calidad de ciudadanos. En este sentido, la enseñanza del derecho lleva consigo una gran responsabilidad, pues exige estimular la imaginación jurídica y moral de los estudiantes y graduados para que puedan establecer comparaciones entre la actividad judicial y las vivencias sociales que permitan resolver cualquier tipo de conflictos. Esta responsabilidad va acompañada del interés por formar profesionales del derecho que actúan con justicia en contextos de diversidad cultural, racial, ideológica o de cualquier otro tipo.

Las apuestas por la justicia social y defensa de los derechos humanos se materializan en las acciones llevadas a cabo en el campo laboral. "La educación superior es un proyecto social que no termina con la educación y la formación profesional. Necesita que los egresados, se aseguren una oportunidad para desempeñar su formación de forma productiva. Es decir, que se organice el proceso educativo para la transición de los egresados al mundo del trabajo, la transición a la vida activa" (Campos Mesa, 2008, p. 326). Sin embargo, sería un error creer que es una tarea fácil. Al igual que sucede en otros planos de la vida individual y social, hay dificultades para mantenerse incólume en un ambiente que puede tornarse hostil, tanto por los obstáculos que lleva consigo la concretización de las teorías como por las contiendas que se libran en el campo laboral por cuenta de la competencia y, en el caso de los recién graduados, la ausencia de experiencias que permitan visualizar de una manera más ágil y estratégica las soluciones a las necesidades del ser humano en medio de contextos de pobreza extrema, migración masiva, desigualdad social. La misma formación debe insistir en que las discusiones, los conflictos y batallas hacen parte de la vida y que no van a cejar, porque son inherentes a la condición humana.

Hacer seguimiento a los graduados de derecho, entonces, puede dar cuenta a su vez de varias aristas: calidad de la formación, actividad del cuerpo docente, pertinencia y adecuación del currículo, aplicación y las dificultades de la puesta en marcha de los preceptos asociados a su profesión y situación laboral. Este último punto conduce a reflexionar sobre la capacidad de movilidad social asociada con la consecución de un título profesional.

Incorporarse al mundo del trabajo, luego de un proceso de formación, suele conllevar al mejoramiento de la calidad de vida. De hecho, la educación es un referente de transformación social que puede cambiar la vida de los graduados y del entorno social en el cual habitan y laboran. Se dice que el siglo XXI es el siglo de las sociedades del conocimiento o de la información: “la principal característica en las relaciones humanas ya no son los productos, ni los servicios, sino el conocimiento. Este constituye el valor agregado fundamental en todos los procesos. Atendiendo a las diferentes etapas del desarrollo histórico de la sociedad y a su relación con el conocimiento" (Sánchez, 2016, p. 237); “el término 'la sociedad de la información', que fue ideado no sólo para reconocer el alcance cada vez mayor de la tecnología avanzada y los sectores con un alto nivel de conocimientos en la economía, sino también destaca que la organización laboral está cambiando como consecuencia de la creciente importancia de los conocimientos" (Red GRADUA2 y Asociación, 2006, p.13). Así que la formación que supone el proceso educativo actual es esencial para el desenvolvimiento en la vida profesional, laboral, social, cultural y política.

En la Conferencia Mundial sobre la Educación Superior - La Educación Superior en el siglo XXI: Visión y acción, realizada por la Unesco en 1998, se expresa la necesidad de reforzar la conexión entre el mundo del trabajo y la previsión de las necesidades de la sociedad. 


\begin{abstract}
Las instituciones de educación superior deberán tomar en consideración sistemáticamente las tendencias que se dan en el mundo y laboral en los sectores científicos, tecnológicos y económicos. A fin de satisfacer las demandas planteadas en el ámbito del trabajo los sistemas de educación superior y el mundo del trabajo deben crear y evaluar conjuntamente modalidades de aprendizaje, programas de transición y programas de evaluación y reconocimiento previos de los conocimientos adquiridos, que integren la teoría y la formación en el empleo. En el marco de su función prospectiva, las instituciones de educación superior podrían contribuir a fomentar la creación de empleos, sin que éste sea el único fin en sí. (Unesco, 1998, p. 8)
\end{abstract}

De ahí que sea conveniente preguntarse por la pertinencia social y laboral al momento de crear nuevos programas y evaluar los existentes, pues, hacia afuera se respondería efectivamente a las demandas del medio a través de la formación de profesionales competentes para comprender, aportar e innovar en la sociedad y hacia dentro se daría cumplimiento a las apuestas y proyectos personales. Dicho de otro modo, no solo se trata de responder, sino de establecer una relación multilateral en la que se reconoce los requerimientos por parte del medio y la capacidad de incidir y transformar el contexto y la propia vida.

A manera de síntesis, con el seguimiento a graduados de la Facultad de Derecho de la Universidad San Buenaventura, Medellín, se busca conocer su situación sociodemográfica, profesional y su participación en distintos escenarios sociales, incluida la relación con la misma institución, con el fin de proponer estrategias que procuren el fortalecimiento de la relación graduados-universidad y mejoren la calidad del programa.

\title{
II. Método
}

Se realizó un estudio de diseño multimétodo (Polit y Hungler, 2005), es decir, se amparó en información de carácter cuantitativa y cualitativa. Desde el punto de vista cuantitativo fue una investigación no experimental descriptiva, es decir, no hubo manipulación de variables independientes y el objetivo básico de la misma consistió en explorar, describir y caracterizar el perfil del graduado de la Facultad de Derecho de la Universidad San Buenaventura. Desde el punto de vista cualitativo se tuvo el apoyo de diferentes herramientas de este tipo de investigación (Rodríguez et al, 1996) lo que permitió comprender e ilustrar la opinión de los graduados frente a la calidad de su formación y los aportes derivados de ella en su vida profesional. La población estuvo constituida por todos los graduados de la Facultad de Derecho desde la primera cohorte hasta el año 2018; la muestra fue de tipo no probabilístico con los graduados que decidieron participar voluntariamente y respondieron la encuesta de forma anónima, una vez aclarado que se realizaría procesamiento estadístico de los datos recolectados y que la participación no tendría ninguna repercusión. También se realizó encuesta al director del programa de pregrado de la Facultad de Derecho.

Dentro de la investigación cuantitativa se consideró las siguientes variables de caracterización a) Características personales: género, edad, lugar de residencia, estrato social, competencias personales. b) Características laborales: condiciones laborales iniciales y actuales: salario, tipo de contrato, área de desempeño, tipo de empresa, reconocimientos y antigüedad. De igual manera dentro de las variables de evaluación de impacto de los graduados se consideró: a) Incidencias académicas e investigativas: publicaciones, reconocimientos académicos, investigaciones, nivel académico, participación en redes, participación en centros de investigación, segundo idioma, obtención de patentes. b) Características sociales, políticas, culturales y artísticas: premios, proyectos, diseño de políticas, cargos públicos o en ONG, relaciones internacionales, participación en organizaciones y redes sociopolíticas, culturales y artísticas.

c) Características empresariales: Iniciativas empresariales, desempeño profesional, reconocimiento 
empresarial. d) Características relacionadas con la formación: grado de correspondencia con los ejes pedagógicos, antropológicos y psicológicos de la Paideia Franciscana, capacidad profesional.

Para la recolección de información cualitativa se usó la técnica de grupos focales para lo cual los graduados fueron citados en las instalaciones de la universidad y la información se recolectó a partir de grabaciones de audio; para el caso de los datos cuantitativos, se aplicó una encuesta para el perfil sociodemográfico y se diseñaron dos cuestionarios, uno sobre competencias profesionales y otro sobre participación en actividades de la universidad. El primer cuestionario presentó un valor adecuado en el modelo de alfa de Cronbach de 0,74, en el segundo instrumento el valor de la fiabilidad en modelo Kuder de Richarson (KR20), obtuvo un valor aceptable de 0,66.

Para el procesamiento de la información, los datos cualitativos se analizaron en Atlas-Ti 7.0; y la información cuantitativa se analizó en el software SPSS versión 23. Para la presentación de las variables del perfil sociodemográfico se usaron tablas de frecuencia y como principal estadístico la moda. En las variables cuantitativas se evaluó la normalidad a partir de la prueba Kolmogorov - Smirnof ( $n>50)$, lo que determinó el uso de estadísticos paramétricos o no paramétricos en el análisis descriptivo e inferencial.

En esta investigación se tuvieron en cuenta las consideraciones éticas emanadas desde la resolución 08430 de 1993 (MSN), acerca de la investigación en seres humanos, sus protocolos e instrumentos fueron sometidos a revisión por parte del Comité de Bioética de la Universidad San Buenaventura, Medellín, la cual otorgó el respectivo aval.

\section{Resultados}

A continuación, se muestran los resultados que permiten caracterizar sociodemográfica y profesionalmente a los graduados de la Facultad de Derecho y dar cuenta de su participación en distintos escenarios sociales, incluida la misma institución, a partir de 56 graduados encuestados, de los cuales $80,4 \%$ son graduados del programa de pregrado, $14,3 \%$ son graduados de alguna de las especializaciones de la Facultad y $5,4 \%$ son graduados de dos o más programas. También se incluyen un aparte en el que se expone la opinión del director de pregrado de la Facultad de Derecho.

\section{Descripción sociodemográfica}

De 56 graduados encuestados, $55,4 \%$ son mujeres y $44.6 \%$ son hombres. Es un dato interesante porque muestra la presencia de mujeres en las disciplinas jurídicas, que históricamente han sido estudiadas por hombres. De hecho, en un porcentaje un poco más alto.

Todos los encuestados viven en Colombia y un gran porcentaje de ellos, 92\% viven en el departamento de Antioquia; el resto vive disgregados en Atlántico, Chocó, Valle del Cauca y Cundinamarca. Tener procesos de formación y desempeñarse laboralmente en el mismo lugar en el que se vive puede ser visto de manera positiva; al conocer el territorio y los problemas, junto con el sentido de pertenencia que lo acompaña, puede ser un valioso prolegómeno para el desempeño laboral y un contrapeso a las consecuencias sociales e individuales que generan las migraciones masivas asociadas a la globalización, como el desarraigo, el desdibujamiento de proyectos de vida, la desvinculación afectiva con sus propias comunidades y territorios. 
El mayor porcentaje de graduados 37,5\% vive en viviendas clasificadas como estrato tres. Le sigue un $22,4 \%$ que habitan en el estrato cuatro. Esto quiere decir que la mitad de los graduados habita en viviendas que tienen ciertas características y que corresponden a los estratos medio-bajo y medio, de acuerdo con la clasificación del DANE. Los graduados que viven en estratos cinco y seis constituyen el $26,8 \%$ y son los considerados estratos medio-alto y alto, respectivamente. El 1.8\% de los graduados, - una persona- vive en el estrato 1 nivel bajo bajo y el 12.5\% habita viviendas que clasifican en el estrato 2 bajo. En general, se puede afirmar que los graduados de la Facultad de Derecho tienen condiciones óptimas de vivienda, exceptuando a las personas que viven en los estratos 1 y 2 . El tipo de vivienda, los materiales con los que está construido, los equipamientos del entorno revelan características socioeconómicas:

la vivienda no es ni ha sido un simple modo de "albergar"; es un modo de habitar y como tal exige que tenga posibilidades de adaptación personalizada según las distintas formas de vida, históricas y culturales, de sus ocupantes. La vivienda es un medio físico que más allá de tener una significación fisiológica, tiene sentido psicológico y socialhistórico referido a la estética y conforme a diversas razones económicas y de posición social (DANE, p.1).

\section{Competencias profesionales}

De acuerdo con las respuestas de los encuestados sobre sus competencias profesionales, $66,1 \%$ considera que tienen competencias superiores, 32,1\% competencias intermedias y el 1,8\% considera que tiene competencias básicas. En este trabajo, estas competencias están asociadas a una serie de capacidades que apuntan a señalar habilidades generales para asumir los retos que supone habitar en las sociedades del siglo XXI. De acuerdo con sus respuestas, 35,7\% considera que tiene un nivel bueno, 32,1\% un excelente, $28,6 \%$ regular, 1,8\% muy deficiente y 1,8\% deficiente capacidad de resolución asertiva de conflictos, de comunicación y trabajo en equipo. Respecto a la capacidad de un manejo eficiente y eficaz de la información y las Tecnologías de la Información y la Comunicación 39,3\% dijo que su nivel era bueno, 26,8\% regular, 17,9\% excelente, $14,3 \%$ deficiente y $1,8 \%$ muy deficiente. Respecto a la capacidad de creación e innovación, el $41,1 \%$ dijo que su nivel era bueno, $26,8 \%$ excelente, $23,2 \%$ regular y $8,9 \%$ deficiente. Respecto a la capacidad de actualización, construcción y uso de nuevos conocimientos $50 \%$ dijo que su nivel es bueno, 26,8\% excelente, $17,9 \%$ regular, 3,6\% deficiente y 1,8\% muy deficiente. Finalmente, respecto a la capacidad de reconocer y respetar las diferencias y de contribuir a una convivencia cuidadosa de los otros $46,4 \%$ dijo que su nivel es excelente, $41,1 \%$ bueno y $12,5 \%$ regular.

También estas competencias profesionales pueden observarse en las producciones académicas, situación laboral, nivel de desempeño en la empresa u organización, rango, trabajo en el área de formación, nivel de ingresos y habilidades comunicacionales en otro idioma. Es decir que la percepción sobre la formación recibida puede constatarse con criterios objetivos que le den sustento a dicha autovaloración.

Resultado del trabajo intelectual de este conglomerado de graduados se han publicado los siguientes productos: 3 artículos, 6 libros, 2 capítulos de libros y 3 líneas de base para el diseño de políticas públicas.

De 56 graduados encuestados, 50\% está empleado y 30,4\% trabaja de manera independiente. Solo el $5,4 \%$, es decir, tres graduados se encuentran desempleados y explican que la causa de su situación laboral se debe a factores relacionados con la ausencia de "aliados y relaciones" y a la exigencia por parte del posible empleador de un nivel educativo superior, específicamente de estudios de maestría. Otro 5,4\% respondió que eran emprendedores; una categoría bastante difusa que necesita ser problematizada tanto 
por la universidad como por los graduados. $8,9 \%$ trabaja como empleado e independiente. En suma, y contando a los emprendedores, $94,6 \%$ de los graduados tienen empleo.

De este porcentaje de graduados con empleo, 51,8\% trabaja en organizaciones de carácter público; $35,7 \%$ en organizaciones privadas; $1,8 \%$ en organizaciones de naturaleza mixta y el $5,4 \%$ no sabe o no responde acerca de la naturaleza de la organización a la cual se encuentra vinculado. Estos datos señalan que los graduados siguen una tradición laboral, propia de los profesionales del derecho, de vinculación con la institucionalidad, lo cual podría significar el mantenimiento de una apuesta por la defensa del Estado; sin embargo, es significativo el porcentaje que trabaja en el sector privado.

La actividad principal de las empresas y organizaciones donde laboran los graduados es Jurídica 28,6\%; Gestión pública 19,6\%; Educación 16,1\%; Otro 8,9\%; consultoría 7,1\%; comercial 5,4\%; Social, cultural, deportiva o artística 3,6\%; Agropecuaria 1,8\%; Tecnologías de Información y Comunicación 1,8\% y Transporte 1,8\%.

Los graduados ocupan cargos en las siguientes áreas: administrativa 23,2\%; de Apoyo y soporte profesional 19,6\%; Docencia 16,1\%; Dirección y gerencia 10,7\%; Otro 10,7\%; Consultoría 8,9\%; Investigación 3,6\%; Desarrollo social, cultural, artístico y deportivo 1,8\%. Dentro de este conjunto, $80,4 \%$ afirma que trabaja en el área de formación recibida y 19,6\% afirma que no. A su vez, de los encuestados que trabajan en su área de formación, 25\% lo hace antes de haberse graduado; 14,3\% inmediatamente al graduarse; 19, 6 \% antes de un año y $16,1 \%$ después de un año; $14,3 \%$ no respondió.

Respecto a la forma de vinculación laboral se tiene que: $33,9 \%$ tiene contrato laboral a término indefinido; $26,8 \%$ tiene otro tipo de vinculación; $16,1 \%$ tiene contrato administrativo de prestación de servicios; $10,7 \%$ Contrato laboral a término fijo; $5,4 \%$ no sabe; $1,8 \%$ por obra o labor. Estas cifras reflejan el contexto de precarización y flexibilidad que caracteriza el ambiente laboral en Colombia. Los graduados del derecho posiblemente tengan más posibilidades de emplearse, sin embargo, comparten las mismas situaciones de inestabilidad que los graduados de otras profesiones. Probablemente, una de las diferencias entre graduados de una y otra sea el tiempo de paro.

Dentro de las competencias profesionales está la competencia en un segundo idioma, que merece una especial atención por cuenta de la apuesta de la Universidad San Buenaventura Medellín por la enseñanza de un segundo idioma que robustezca la formación profesional de sus estudiantes, de acuerdo con los lineamientos del Ministerio de Educación Nacional. De acuerdo con la respuesta, $57.1 \%$ considera que tiene un nivel básico, 33.9\% un nivel intermedio y 8,9\% tiene un nivel superior. Si se disgrega en las habilidades asociadas a las competencias en un segundo idioma, se observa que en escritura $57,1 \%$ dijo que su nivel era bajo, $33,9 \%$ medio y $8,9 \%$ alto. Respecto a la capacidad de sostener una conversación $66,1 \%$ dijo que su nivel es bajo, $26,8 \%$ nivel medio y $7,1 \%$ nivel alto. Con la competencia lectora los resultados son levemente distintos: $46,4 \%$ tiene un nivel lectura medio, $42,9 \%$ bajo y $10,7 \%$ alto. Estos datos señalan que las habilidades comunicacionales en otro idioma son deficientes. Si los graduados aspiran a trabajar en otros lugares del mundo este es un requisito indispensable.

\section{Relaciones con la comunidad}

De acuerdo con sus respuestas, en general, los graduados mantienen un vínculo muy tenue con la institución. 92,9\% de los encuestados afirmó que su relación es básica; $48,2 \%$ respondió que su forma de participación es a través del diligenciamiento de sondeos y encuestas. El uso de escenarios deportivos o culturales y de los servicios de librería, biblioteca y laboratorios es casi nulo: $96,4 \%$ y $91,1 \%$ de los graduados respectivamente 
respondió que no hacían uso de ellos. Respecto a la asistencia a reuniones, llamados y capacitaciones de actualización laboral la respuesta de los graduados es mayoritariamente negativa: 89,3\% dijeron que no asistían y 10,7\% dijo que sí lo hacía. Solo una persona, 1,8\%, promueve la vinculación de practicantes y profesionales de la USB y seis personas, 10,7\%, participan en las redes sociales de la comunidad. A lo que se refiere propiamente a actividades de índole académico, como asistencia o ponencia en cátedras abiertas y continuación de estudios, el porcentaje negativo también es mayoritario. Más del $90 \%$ respondió que no se vinculaba por estos medios.

Los graduados de la Facultad de Derecho han tenido reconocimientos públicos en las siguientes áreas: científicas/académicas (4), artísticas (1), laboral (3), social (1), político (1) empresarial (1). De carácter institucional, municipal, nacional e internacional (7).

De acuerdo con las respuestas dadas por el director de pregrado de Derecho, las fortalezas son la formación humanística-franciscana, la formación socio jurídica, la formación para la conciliación mediante la resolución alternativa de conflictos y la apuesta por la investigación. El aspecto que se podría mejorar es seguir insistiendo en la virtualización del proceso educativo. Según el director, los graduados de la Facultad no tienen mayores problemas de empleabilidad, pues son reconocidos y acogidos en el medio debido a su formación en Derechos Humanos, Derecho Público, Derecho Penal, Mecanismos alternativos de resolución de conflictos, entre otras.

En el desarrollo de este trabajo de seguimiento a graduados en el que se buscó, en términos generales, conocer su situación profesional y sociodemográfica, el estado del vínculo con la institución y la comunidad, se presentaron ciertas limitantes, como el número de la muestra y el tiempo suficiente para aplicar otro tipo de instrumentos de investigación que profundizaran correlaciones entre variables y que permitieran conocer la percepción de un número significativo de empleadores. Además, hubiese sido enriquecedor contar con más puntos de vista de las directivas relacionadas con la Facultad. Sin embargo, estos resultados son un insumo significativo para conocer la intervención de los graduados en distintos escenarios, el estado de sus competencias profesionales y establecer su perfil sociodemográfico y proponer formas más eficaces de seguimiento.

\section{Discusiones}

En este apartado se discutirán los siguientes puntos de acuerdo con resultados obtenidos de la caracterización sociodemográfica, las competencias profesionales y la relación con la comunidad universitaria y sociedad y la producción teórica al respecto.

Al igual que sucede en otras facultades de Derecho del país y también en otras carreras, las mujeres componen la mitad o incluso un poco más del grupo de estudiantes. En el campo del derecho, la presencia de las mujeres podría darle una nueva orientación a la forma de resolver los conflictos jurídicos, pues, “abogados y abogadas aportan distintas miradas sobre las problemáticas jurídicas y en particular sobre temas como los referidos al derecho de familia [...] a través de la incorporación de una nueva perspectiva que seguramente conducirá a modificaciones graduales de nuestras instituciones jurídicas" (Suárez, 2012, p. 181). Se podría ver en la presencia de las mujeres un indicador de la transformación hacia una sociedad más democrática e incluyente y de cambios dentro de la misma jurisprudencia. Sin embargo, es importante relacionarla con otras variables como cargo ocupado, ingresos y nivel académico, incluso con la composición de los currículos, con el fin de ampliar los horizontes de interpretación. 
Pese a que más personas, hombres y mujeres, acceden a educación universitaria que implicaría una mayor movilidad social y mejoramiento de la calidad de vida, de acuerdo con la cifra de ingresos y la ubicación en estratos socioeconómicos medios y bajos, se observa que titularse no garantiza la obtención de unos ingresos altos, aun en una profesión como el derecho que tiene tanto prestigio social. Según García Villegas y Ceballos Bedoya (2019),

Las diferencias de clase de los pregrados se reproducen luego en el ejercicio profesional. Los cargos con remuneraciones bajas o medias están copados por los graduados de pregrados de bajo costo y menor calidad, que suelen ser los juristas de clase media o baja. Por el contrario, en los cargos más altos tiende a haber un predominio de los graduados de las mejores universidades privadas, que suelen ser los más ricos. Así se configura una especie de apartheid educativo que se traslada al mundo profesional, donde las clases altas obtienen los mejores cargos y las clases bajas los peores. Esta situación socialmente discriminatoria empieza desde mucho antes, en la educación básica, entre colegios de buena calidad que reciben a los hijos de la clase alta y colegios mediocres que reciben al resto de la población (p. 20).

Sin embargo, reconociendo que en Colombia las clases sociales se reproducen a través de múltiples mecanismos, incluidos los provenientes del sistema educativo, es necesario señalar que hay movilidad social hacia arriba, eso sí, no tanto como en otros países latinoamericanos. La movilidad social ascendente que se deriva de la educación, que es uno de sus desencadenantes, no el único, es baja pero estable. De acuerdo con Ángulo y los demás (2012), las desigualdades sociales en Colombia se mantienen, aun teniendo en cuenta el progreso educativo. En sus palabras: "el progreso educativo ha sido insuficiente para reducir las diferencias iniciales con otros países latinoamericanos" (p. 22). De ello se colige que la educación no es garantía para lograr ascenso social; por mucho que la idea, según la cual obtener un título universitario es pase infalible para tener mejores condiciones de vida, persista; las cifras de desempleo y pobreza lo demuestran. Ahora bien, en lo que respecta al Derecho, probablemente la obtención de educación posgradual influya en el aumento de ingresos. El reto de las IES es grande y claramente no depende de ellas solamente; debe haber una articulación con el Estado y con el sector productivo que procure el beneficio de toda la sociedad y que genere una revisión de sus postulados sobre las formas existir y trabajar que permitan vivir dignamente.

\section{Competencias profesionales del graduado de Derecho}

La educación superior universitaria en Colombia, al igual que otros países en Latinoamérica, ha incorporado el enfoque de competencias en la formación de sus distintos programas, pese a los cuestionamientos que aún suscita (Aedo Barrena, 2014, p. 105). De hecho, el Ministerio de Educación adoptó tal modelo para concebir el sistema educativo en Colombia. Sin embargo, aunque está establecida, es importante tener presente que se trata no solo de algo puramente académico, sino que, como afirma Clavijo (2015): "en realidad el asunto tiene una dimensión política y fuertemente ideológica [...] ¿ ipor qué asumir el enfoque por competencias y no otro enfoque, el tradicional por ejemplo? ¿Qué implicaciones conlleva la implementación de este modelo? pero además ¿Quién decide cuáles son las competencias para formar en cada programa o área de conocimiento?" (p. 188).

En todo caso, las competencias en la educación superior permitirán, de acuerdo con Tobón, citado por Clavijo (2015, p. 190), establecer una relación más estrecha entre la universidad y la empresa, por cuenta de una adecuación de la formación a las demandas de las organizaciones. Es muy común hacer referencia 
al choque que genera ingresar al mundo laboral; pareciera que teorías y conocimientos aprendidos no encontraran cauce en la vida laboral, sobre todo, en disciplinas liberales y humanísticas. Pues bien, se supone que las competencias facilitarían dicho engranaje. Además, permitirían reconocer y potenciar la pertinencia de acuerdo con el contexto social. Por otra parte, las competencias permitirían la movilidad de los agentes de la comunidad universitaria, estudiantes, graduados, docentes, por cuenta del proceso de "homologación y transferencia de la formación" (Clavijo, 2015, p. 190). En suma, el enfoque de competencias permite estar a tono con el resto del mundo.

En lo que respecta propiamente a las competencias profesionales del abogado, al igual que en las otras profesiones, hay discusiones sobre cuáles deben ser, es decir que no hay una sola forma de caracterizar cómo sería un graduado ejemplar de derecho. Una de las razones estriba en los proyectos, misión y visión particulares de cada institución universitaria y su forma de articularse con la sociedad. Pese a estos sanos desacuerdos, en general, el graduado de derecho se forma en competencias específicas propias de su profesión, por más que pese la imprenta de la institución desde la cual es graduado.

Según la resolución 2768 de 2003 del Ministerio de Educación Nacional, los graduados de Derecho deberán tener "[...] competencias cognitivas, investigativas, interpretativas, argumentativas y comunicativas, así como capacidades para la conciliación, el litigio y para el trabajo interdisciplinario" (Art. 2), relacionadas con las siguientes características:

- Una sólida formación jurídica, humanística y ética, que garantice un ejercicio profesional en beneficio de la sociedad;

- Capacidad analítica y crítica para la interpretación de los problemas sociales, políticos y económicos del país, así como del impacto de las normas frente a la realidad.

- La plena conciencia del papel mediador y facilitador que cumple el abogado en la resolución de conflictos.

- Suficiente formación para la interpretación de las corrientes de pensamiento jurídico.

- El desarrollo de habilidades comunicativas básicas en una segunda lengua.

- Las demás características propias de la formación que se imparte en la institución de acuerdo con su misión y proyecto institucional y con la tradición universal del conocimiento jurídico. (Art.2)

Como se observa, estas disposiciones resaltan la importancia de la imbricación con el contexto social del país, aunque una de las tendencias del derecho a nivel mundial sea aspirar a una formación que permita desenvolverse en la llamada "aldea global", en la cual los problemas más acuciantes están relacionados con asuntos de bioética y genética, tecnología y comunicaciones, protección del medio ambiente y relaciones comerciales trasnacionales (Clavijo, 2015, p. 198). Sobre todo, estos dos últimos puntos son apremiantes en nuestro país, por ejemplo, por cuenta de la diversidad natural y la firma de tratados comerciales internacionales.

Dadas las condiciones generadas por la pandemia del coronavirus, hay que agregar a esta lista de premuras la defensa de los derechos digitales y, paralelamente, el derecho a la privacidad de los ciudadanos. Además, hay que anotar que Colombia es un país con problemas sociales de gran envergadura asociados con el conflicto interno y a la desigualdad estructural, que reclaman la presencia de abogados y abogadas comprometidos con la resolución de conflictos y defensa de los derechos humanos. La mirada humanística que guía el proyecto misional de la Universidad San Buenaventura, Medellín, en nuestro contexto es, sin dudas, una base que orienta el quehacer profesional de los graduados atendiendo a estas necesidades y urgencias. 
Ahora bien, es necesario ver las formas en que estas intenciones se materializan en el campo laboral y se relacionan con el contexto social e histórico colombiano. Hay ciertas circunstancias que particularizan la situación laboral de los graduados de derecho. Primero, dada la relevancia que tiene la profesión, por cuenta de su capacidad de intervenir en la realidad social desde la normatividad, es un punto de atención los cargos ocupados por los graduados. Hay cargos de altísima responsabilidad e incidencia nacional que son disputados por graduados de distintas universidades de élite. La reproducción social de los privilegios de clase también atañe a los graduados de las ciencias jurídicas.

En segundo lugar, el nivel de desempleo de los graduados de derecho es relativamente bajo en comparación con otras carreras, como sociología o historia, contando con que el número de graduados de derecho es bastante considerable. La razón radica en que una vez graduado, el profesional en derecho puede litigar sin tener que certificar experiencias y dado que Colombia es un país legalista que tiende a resolver sus conflictos apelando a alguna autoridad judicial, pues invariablemente habrá casos que necesitarán ser resueltos a través de asesorías de todo tipo. "En Colombia (también en América Latina) los abogados litigantes son y han sido los protagonistas de la profesión jurídica." (García Villegas y Ceballos Bedoya, 2019, p. 16), aunque según anota Clavijo (2015) "el litigio está pasando a un segundo plano; el mundo está formando abogados conciliadores, asesores, constructores de desarrollo, demoledores de fronteras, profesionales que tienen claridad sobre las necesidades jurídicas en la construcción de un nuevo orden mundial" (pp. 200-201). Parece que Colombia no se adherido suficientemente a esta tendencia.

El asunto con el litigio es que puede ser el punto de inicio y de llegada de los profesionales del derecho; un ir y venir entre el sector público y privado que afectaría la salud de la justicia. En el marco del sistema neoliberal, hay embates contra la funcionalidad del Estado a favor de la libertad del mercado. Según García Villegas y Ceballos Bedoya (2019):

En Colombia, al igual que en muchos otros países de América Latina, la profesión jurídica está moldeada por el mercado. Este es, además un fenómeno que se ha acentuado con la globalización de la economía y con el papel central que los abogados litigantes afiliados a los grandes bufetes en cada país están teniendo en la configuración del Estado, del mercado y del campo jurídico en las sociedades contemporáneas (p. 213).

En este mismo sentido, Cardona Zuleta y Montoya Giraldo (2019) argumentan que el mercado puede llegar a tal punto de influir en la educación que provocaría "tomar decisiones político-administrativas y académicas en detrimento de la calidad, rigurosidad y pluralidad de los contenidos de las diferentes materias que componen los planes de estudio [...] situación que en el campo jurídico es bastante compleja porque llevaría a una mayor compartimentación y fraccionamiento de los conocimientos en contraposición a las ideas de flexibilidad e interdisciplinariedad curricular" (p. 290). Aun reconociendo el estado de la situación, de cualquier modo, la interacción con el sector productivo es inexorable. Habría que ver y buscar el equilibrio de esta correlación de fuerzas entre agentes sociales con igual peso. "Si América Latina ya aprendió que mercados competitivos son indispensables para desatar las energías del crecimiento económico, ahora aprende que ni la igualdad social ni la estabilidad política son bienes que se logran en la economía de mercado" (Proyecto, 2010, p. 41-42).

En el campo del derecho, el contrapeso a dicha situación se sitúa en la formación integral que defiende lo público, la institucionalidad, la protección de los derechos humanos, la conciliación, la resolución alternativa de los conflictos, entre otras. Las facultades de Derecho de la misma manera que incentivan la capacidad de adaptación para enfrentar lo nuevo y desconocido con el fin de adelantar proyectos, interrelacionarse e interactuar en equipos multidisciplinarios -de acuerdo con el espíritu de estos tiempos-, 
deben hacerlo en las formas de moldear la realidad según ciertos principios que le den lugar a la agencia y dignidad de los sujetos.

\begin{abstract}
... la dignidad del ser humano como ser ético, una dignidad plenamente igual e independiente del lugar donde se encuentre ese ser humano; la sociabilidad humana, de acuerdo con la cual una vida con dignidad humana significa una vida en común con otros, organizada de modo que respete aquella igual dignidad; y las múltiples necesidades humanas, las cuales sugieren que esta vida común debe hacer algo por todos nosotros; satisfacer nuestras necesidades hasta un punto en el que la dignidad humana no se vea comprometida por el hambre, la violencia o el trato desigualdad en el espacio político. [...] debemos producir, y habitar, un mundo moralmente decente, un mundo en donde todos los seres humanos tengan lo que necesitan para vivir una vida acorde con la dignidad humana (Nussbaum, 2018, p. 74).
\end{abstract}

\title{
Las Facultades de Derecho y sus graduados
}

Al ser el derecho una carrera con tanto prestigio social suele hacerse una asociación muy rápida entre la universidad de la cual se es graduado y la calidad profesional del graduado. Quizás sea una de las profesiones en las cuales el reconocimiento de la universidad es determinante para atraer a los futuros estudiantes; sin embargo, de acuerdo con Carlos Molina (2017), director ejecutivo de la Asociación Colombiana de Facultades de Derecho, aunque ciertas facultades de derecho de universidades de la capital del país aparecen dentro del listado QS de Derecho para América Latina, sus graduados se encuentran asociados a graves problemas de corrupción. Además, este es uno de los medios para medir la calidad de la formación, no el único, pues se cuenta con información de Colciencias, la Asociación Colombiana de Facultades de Derecho, el Ministerio de Educación Nacional y el Icfes, que pueden medir otras variables.

Molina (2017) destaca un punto importante para los propósitos de este trabajo: las universidades pueden hacer esfuerzos administrativos para cumplir ciertos parámetros con el fin de aparecer en listados clasificatorio, de acuerdo con el análisis de la Revista Semana que trae a colación: "Los ranking siempre resultan subjetivos: son incapaces de medir cada una de las facetas de la vida académica, y además, las universidades mejor administradas pueden mejorar sus resultados al enfocar sus esfuerzos en los criterios de la clasificación. [...] seguir cada una de las mediciones, sin necesariamente ofrecer una mejor formación académica" (s.f.). La crítica se dirige a la minusvaloración de la formación ética de los estudiantes en el proceso de profesionalización. Si las facultades de Derecho le apostarán a la formación en valores y defensa de lo público sería en efecto una formación integral en el participarían activamente todos los miembros de la comunidad educativa.

En este sentido no solo se trata de medir la calidad a partir de la pertinencia curricular, que por cierto se le pueden hacer numerosas críticas; “la educación que se ofrece en las facultades de derecho [como] una propuesta educativa fundamentalmente formalista, aparentemente racional y neutral, que reproduce la ideología de la visión liberal del Estado" (Gómez Sánchez, 2016, p. 234), sino de incorporar otros elementos para una evaluación global de la calidad.

En el caso del derecho, como bien afirma García Villegas y Ceballos Bedoya (2019), el mejoramiento de la calidad va de la mano de la estimulación de una cultura jurídica, defensora de la institucionalidad, de la Constitución y de los derechos fundamentales, que se “inculca en las facultades de Derecho, se promueve por parte de protagonistas del campo jurídico (profesores prestigiosos, magistrados de las altas 
Cortes, decanos y abogados de prestigio) y se controla desde las instituciones, con sanción para quienes contravengan las normas jurídicas y sobre todo éticas que regulan la profesión" (p. 24).

\section{Conclusiones}

Para estimar la calidad de las IES y particularmente de las facultades de las universidades hay que tener en cuenta varios criterios. Uno de ellos es el resultado interpretativo de proyectos de seguimiento a graduados, porque en ellos se reúnen las apuestas institucionales, tanto a nivel formativo como misional, y la presencia del sector productivo y la sociedad civil. Las respuestas a los cuestionarios fueron reunidas en tres grandes grupos: perfil sociodemográfico, competencias laborales y relación con la institucionalidad, que pueden ser analizadas de manera transversal, como partes de un todo y con múltiples relaciones entre sí.

Del perfil sociodemográfico de los graduados de la Facultad de Derecho se puede inferir que la mayoría pertenece a estratos socioeconómicos medios. Posiblemente acceder al mercado laboral haya influido en tal situación; no en vano dentro del seguimiento a graduados es irremplazable atender a su situación laboral, pues es indicio de calidad y pertinencia del programa y de la probidad de la universidad que lo desarrolla. Como lo revelan los resultados, la mayoría de las personas graduadas de la Facultad de Derecho de la USB, Medellín, están empleadas, a pesar del contexto laboral en Colombia, que se caracteriza por la precariedad, inestabilidad y flexibilidad, lo cual demuestra la pertinencia del programa. Sin embargo, por esta misma razón, hay que tener en la cuenta el tipo de cargos ocupados y el nivel de responsabilidad asociados a ello. Para estudios de seguimiento ulteriores, la intersección entre las variables de género, estrato social y cargo podría dar otra serie de elementos para profundizar en el perfil sociodemográfico de los graduados.

El solo hecho de conocer que hay una gran población de mujeres juristas, como lo son las graduadas de la Facultad de Derecho de la San Buenaventura, Medellín, es motivo de satisfacción, por cuenta de tener conciencia de que muchos años atrás la situación era diferente, por muchas razones, entre ellas la configuración de una sólida estructura social patriarcal que confinaba a las mujeres a las labores domésticas. Sin embargo, si estos datos de distribución sexual de los graduados se comparan con datos de rango salarial, se podría obtener información valiosa que refute o confirme que la inserción de mujeres profesionales en el mercado laboral incide en la configuración de los salarios de ambos, hombres y mujeres.

Es muy común escuchar que Colombia es un país de abogados y abogadas; el cuantioso número de ellos y la relevancia y reconocimiento social, que otras profesiones envidiarían, lo confirman. Este "es un fenómeno que tiene muchas causas, entre ellas la urbanización, la globalización económica, la expansión territorial de la oferta de pregrados de Derecho, la privatización del Estado y las complejidades jurídicas derivadas de este hecho, etc." (García Villegas y Ceballos Bedoya, 2019, p.19). En este contexto es que se enmarca una suerte de competitividad entre las distintas universidades que tienen programas de derecho. Poco a poco, y con mucha insistencia y trabajo se ha logrado descentralizar la profesión de la abogacía, aunque es innegable que los altos cargos asociados con la profesión, como ministerios, están ocupados por graduados de las universidades de élite ubicadas en Bogotá, la capital del país.

La contrapartida a este centralismo y jerarquización es la constitución y mantenimiento de universidades con niveles altos de calidad en los otros centros administrativos, económicos y políticos del país. La calidad radicaría en la composición del currículo, atención al contexto social, la formación docente, instalaciones físicas adecuadas, un conjunto de principios misionales, entre otros. Dado que hay tantas universidades e 
institutos universitarios, públicos y privados, ofreciendo programas de derecho es necesario resaltar las particularidades de cada cual. En el caso de la Facultad de Derecho de la Universidad San Buenaventura, la vocación humanística franciscana, la formación en Derechos Humanos, la enseñanza de formas alternativas de resolución de conflictos, le dan un sello particular que influiría en la forma de establecer relaciones con la sociedad y con el sector productivo.

También hay que tener presente que, para estimar la calidad de un programa de derecho, sea de pregrado o de posgrado, es necesario incluir criterios que complementen aquellos derivados de la relación con el mercado. Si bien, es importante que los graduados estén empleados en su área de formación, también lo es que ejerzan su profesión de una manera ética y desarrollando las habilidades y competencias específicas del derecho. Junto con los demás actores de la comunidad universitaria las IES pueden emprender acciones que mejoren la calidad de los programas.

Actualmente las tendencias del derecho exigen a sus profesionales adquirir competencias en un segundo idioma, puesto que la movilidad internacional ha sido facilitada por los convenios interuniversitarios y la posibilidad de viajar y vivir en otros países ha aumentado y algunos de los temas más acuciantes en el marco del derecho tienen que ver con tratados comerciales internacionales. Igualmente, aprender un segundo idioma permite acceder a datos, informaciones y elucubraciones, no traducidos, que podrían nutrir la reflexión propia. En este sentido, sería prudente seguir fortaleciendo con ahínco el aprendizaje de un segundo idioma en la institución para posibilitar la comunicación en diferentes niveles.

Igualmente se hace necesario insistir en la virtualización, tal como lo sugiere el director del pregrado de Derecho. Habrá que preguntarse con qué fin, cuáles serían sus beneficios, cuáles sus tropiezos, cómo se combinaría con la necesaria e indispensable presencialidad, entre otras cuestiones que surgirán si se consideran variables de espacio, tiempo, ruralidad, costos, conectividad, pedagogía, etc. Es un asunto que no se puede ignorar porque hace parte de la actualidad del mundo.

Por otra parte, es conveniente fortalecer el sistema de información sobre el desempeño profesional, a nivel laboral, social, político y cultural, de los graduados mediante la recuperación del vínculo con la Universidad. De esta manera se tendrá noticias de su influencia en la sociedad y se podrá convocarlos a diferentes actividades que realice el alma máter.

De esta reflexión sobre el seguimiento de los graduados de la Facultad de Derecho de la Universidad San Buenaventura se derivan una serie de reflexiones que pueden ser de interés para los miembros de otras comunidades académicas, tanto para graduados como estudiantes, incluidos directivas y empleadores.

\section{Referencias Bibliográficas}

Aedo Barrena, C. E. (2014). Formación por competencias y enseñanza del Derecho. En Revista Pedagogía Universitaria y Didáctica del Derecho (Santiago de Chile),1(1):104-113. Doi:10.5354/0719-5885.2015.35966

Ángulo, R.; Azevedo, J. P.; Gaviria, A. y Paéz, G. N. (2012). Movilidad social en Colombia. En Serie Documentos Cede 43. Recuperado de: https://economia.uniandes.edu.co/components/com_booklibrary/ebooks/ dcede2012-43.pdf

Berrún Castañón, L. N. (Coord.) (2015). Estudio de seguimiento de egresados de la UANL. México: Universidad Autónoma de Nuevo León.

Campos Mesa, M. (2008). Los egresados y su inserción laboral ¿Estudias o trabajas? En Revista Fuentes. Sevilla (España), 8, 322-332. Recuperado de https://revistascientificas.us.es/index.php/fuentes/ article/view/2531 
Cardona Zuleta, E., \& Montoya Giraldo, A. (2019). Análisis de la Inserción Laboral y la Empleabilidad de los Egresados. ¿Condición de Calidad o imposición que distorsiona los sistemas de medición de calidad de la Educación Superior? En Estudios De Derecho, 76(168): 273-296.

Clavijo Cáceres, D. (2015). El enfoque de competencias en la formación del abogado para el siglo XXI. En Justicia, 27: 185-212. Doi https://doi.org/10.17081/just.20.27.813

Constitución Política de Colombia. (2018). Bogotá: Legis.

DANE (s.f.) Preguntas frecuentes estratificación. Recuperado de https://www.dane.gov.co/files/ geoestadistica/Preguntas_frecuentes_estratificacion.pdf

Decreto 1330/2019. Recuperado de https://www.mineducacion.gov.co/1759/w3-article-387348.html?_ noredirect=1:

Fonseca Ferís, R. (2019). Responsabilidad social universitaria. ¿Una tarea pendiente? En Revista Científica Internacional De La Universidad Tecnológica Intercontinental, Asunción (Paraguay), 6(1): 179-192. Recuperado de http://www.utic.edu.py/revista.ojs/index.php/revista/article/view/89

García Villegas, M y Ceballos Bedoya, M. A. (2019). La profesión jurídica en Colombia. Falta de reglas y exceso de mercado. Bogotá: Ediciones DeJusticia

Gómez Sánchez, G. I. (2016). ¿Abogados para la democracia o para el mercado?: Repensar la educación jurídica. En Revista de Derecho. Universidad del Norte Barranquilla (Colombia), 466: 225-256. Doi http://dx.doi.org/10.14482/dere.46.8817.

Misas Arango, G. (2004). La educación superior en Colombia. Análisis y estrategias para su desarrollo. Bogotá: Universidad Nacional de Colombia.

Molina Betancur, C. M. (2017). Análisis: Facultades de Derecho y corrupción. En Ámbito Jurídico. Recuperado de: https://www.ambitojuridico.com/noticias/academia/educacion-y-cultura/analisis-facultadesde-derecho-y-corrupcion

Nussbaum, M. (2018). Las Capacidades más allá de las Fronteras Nacionales. Bogotá: Editorial Planeta.

Polit, D., \& Hungler, B. (2014). Investigación Científica en Ciencias de la Salud. México: Mcgraw-Hill / Interamericana de México.

Proyecto Educativo Bonaventuriano - PEB (2010). Bogota: Editorial Bonaventuriana. Recuperado de https:// www.usbmed.edu.co/Portals/0/PDF/Doc-Institucionales/PEB-2010.pdf

Red GRADUA2 y Asociación Columbus (2006). Manual de instrumentos y recomendaciones sobre el seguimiento de egresados. México: Instituto Tecnológico y de Estudios Superiores de Monterrey. Recuperado de https://www.almalaurea.it/sites/almalaurea.it/files/docs/universita/altro/red_ gradua2.pdf

Resolución 2768/2003, Art. 2. Recuperado de https://www.mineducacion.gov.co/1759/w3-article-86421. html?_noredirect=1

Sánchez, R. A. (2016). La Sociedad de la Información, Sociedad del Conocimiento y Sociedad del Aprendizaje. Referentes en torno a su formación. En Biblioteca Anales de Investigación, 235-243.

Suaréz, P. S. (2012). Las mujeres abogadas en la historia y en la Facultad de Derecho de la Universidad de Buenos Aires. En Academia. Revista sobre enseñanza del Derecho (Argentina),10(20):143-183. Recuperado de http://www.derecho.uba.ar/publicaciones/rev_academia/revistas/20/las-mujeresabogadas-en-la-historia-y-en-la-facultad-de-derecho-de-la-universidad-de-buenos-aires.pdf

Unesco (1998). Conferencia Mundial sobre Educación Superior - La educación Superior en el siglo XXI. Recuperado de https://unesdoc.unesco.org/ark:/48223/pf0000113878_spa 
Vázquez Olivera, M. G. (2015). La calidad de la educación. Reformas educativas y control social en América Latina. En Latinoamérica. Revista de Estudios Latinoamericanos (México), 1, 93-124. Doi: https://doi. org/10.1016/j.larev.2014.10.001

Osorio Valencia, J. (2017). Prácticas de los abogados en formación en los consultorios jurídico. En Revista Virtual Via Inveniendi et ludicand, 119-148. Doi: https://doi.org/10.15332/s1909-0528.2017.0001.02 